\title{
Fabrication and Testing of 4.2m Off-Axis Aspheric Primary Mirror of Daniel K. Inouye Solar Telescope
}

\author{
Chang Jin Oh *a , Andrew E. Lowman ${ }^{\text {a }}$, Greg A. Smith ${ }^{\text {a }}$, Peng Su ${ }^{\text {a }}$, Run Huang a , Tianquan $\mathrm{Su}^{\text {a }}$, \\ Daewook Kim ${ }^{\text {a }}$, Chunyu Zhao ${ }^{\mathrm{a}}$, Ping Zhou ${ }^{\mathrm{a}}$, James H. Burge ${ }^{\mathrm{a}}$ \\ ${ }^{\text {a }}$ College of Optical Sciences, the University of Arizona \\ 1630 E. University Blvd, Tucson, AZ, 85721, USA
}

\begin{abstract}
Daniel K. Inouye Solar Telescope (formerly known as Advanced Technology Solar Telescope) will be the largest optical solar telescope ever built to provide greatly improved image, spatial and spectral resolution and to collect sufficient light flux of Sun. To meet the requirements of the telescope the design adopted a $4 \mathrm{~m}$ aperture off-axis parabolic primary mirror with challenging specifications of the surface quality including the surface figure, irregularity and BRDF. The mirror has been completed at the College of Optical Sciences in the University of Arizona and it meets every aspect of requirement with margin. In fact this mirror may be the smoothest large mirror ever made.

This paper presents the detail fabrication process and metrology applied to the mirror from the grinding to finish, that include extremely stable hydraulic support, IR and Visible deflectometry, Interferometry and Computer Controlled fabrication process developed at the University of Arizona.
\end{abstract}

Keywords: DKIST, Off-Axis Parabola, Deflectometry, SCOTS, Interferometry, Fabrication, Testing

\section{INTRODUCTION}

The Daniel K. Inouye Solar Telescope will be the largest optical solar telescope ever built and the most powerful solar telescope to observe the Sun and its magnetic activities at higher resolutions. To achieve high resolution and sensitivity, the DKIST adopts $4.2 \mathrm{~m}$ large off-axis parabolic primary mirror. The telescope has a large field of view — up to 3000 arcsec out of an average solar diameter of 1,920 arc-seconds - and will resolve down to 0.03 arcsec in visible light (550 $\mathrm{nm}$ ). This will reveal features as small as 20 to $70 \mathrm{~km}$ wide at the solar surface. ${ }^{1,2} \mathrm{In}$ addition, an off-axis design for the primary was chosen to minimize scattered light and avoid obstructing support structures (which rotate in the image plane) from compromising the adaptive optics. ${ }^{2}$

Figure 1. shows the detail construction of the mirror blank and the calculated aspheric departure of the mirror.
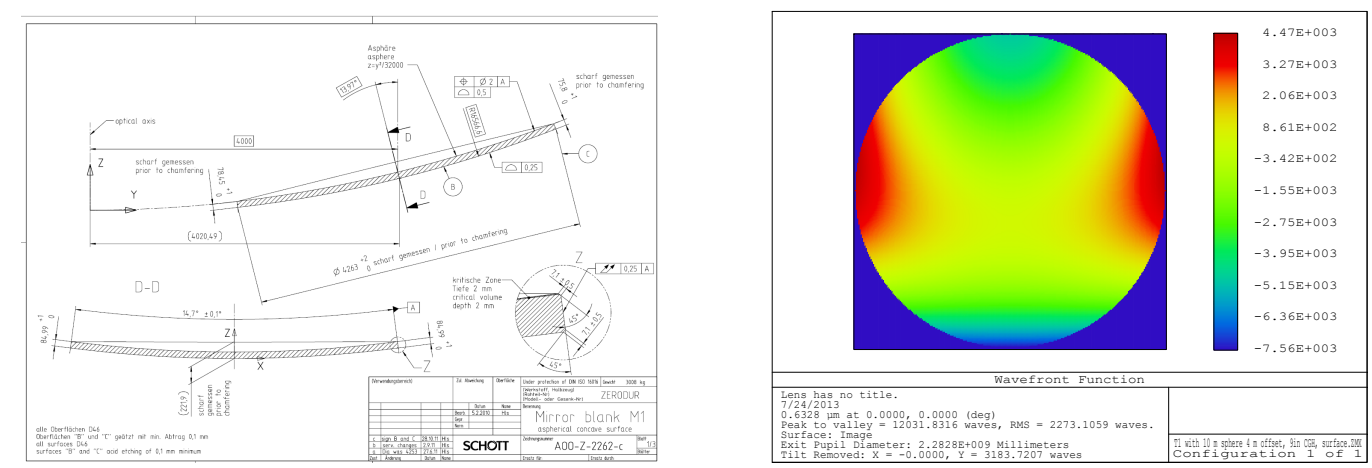

Figure 1. Drawing of the $4.2 \mathrm{~m}$ DKIST primary mirror blank (Left) and the aspheric departure of the mirror (Right)

*cjoh@optics.arizona.edu

Advances in Optical and Mechanical Technologies for Telescopes and Instrumentation II, edited by Ramón Navarro, James H. Burge, Proc. of SPIE Vol. 9912, 991200

(C) 2016 SPIE · CCC code: 0277-786X/16/\$18 - doi: 10.1117/12.2229324 
The mirror blank is constructed as a thin meniscus type for an active mirror support in the telescope. The mirror prescription is shown in Table 1 and as it is described Figure 1 the aspheric departure is as large as approximately $9 \mathrm{~mm}$ $\mathrm{p}-\mathrm{v}$.

Table 1. The prescription of the mirror

\begin{tabular}{cc}
\hline Items & Parameters \\
\hline Material & Zerodur (SCHOTT) \\
Diameter $(\mathrm{mm})$ & 4200 \\
Thickness $(\mathrm{mm})$ & 75 (aspect ratio=1/56) \\
Conic, $\mathrm{K}$ & -1 \\
Radius of Curvature, RoC $(\mathrm{mm})$ & 16000 \\
Off-axis Distance $(\mathrm{mm})$ & 4000 \\
Aspheric Departure $(\mathrm{mm})$ & 9 \\
\hline
\end{tabular}

Table 2 shows the fabrication specification and requirements of the mirror. The DKIST mirror specifically set a requirement for the Bidirectional Reflectance Distribution Function (BRDF) for the science.

Table 2. Specifications and requirements of the mirror

\begin{tabular}{cc}
\hline Items & Parameters \\
\hline Surface Figure (nm RMS) & 25 \\
mid spatial figure (nm RMS) & 8 (over 100 1mm) \\
Surface Roughness (nm RMS) & 2 \\
BRDF (sr' s $^{-1}$ & 1.0 \\
Radius of Curvature, RoC (mm) & \pm 15 \\
Off-axis Distance (mm) & \pm 2 \\
Force Allowance, (N max) & 20 (up to 30 modes) \\
\hline
\end{tabular}

Large aspheric departure makes the fabrication and testing difficult and the requirement for the high smoothness is another challenge. The details of fabrication and testing process have been described in the following sections.

\section{METROLOGY SUPPORT}

The mirror support is a critical element for the fabrication and metrology due to the extreme aspect ratio of the mirror blank construction. The mirror support of the telescope has 119 axial actuators with a set of force distribution to maintain the mirror shape. During the fabrication and testing the mirror is desired to be supported using the same set of force distribution as in the telescope. Additionally the long term stability over entire fabrication period is very important to ensure the high repeatability in metrology.
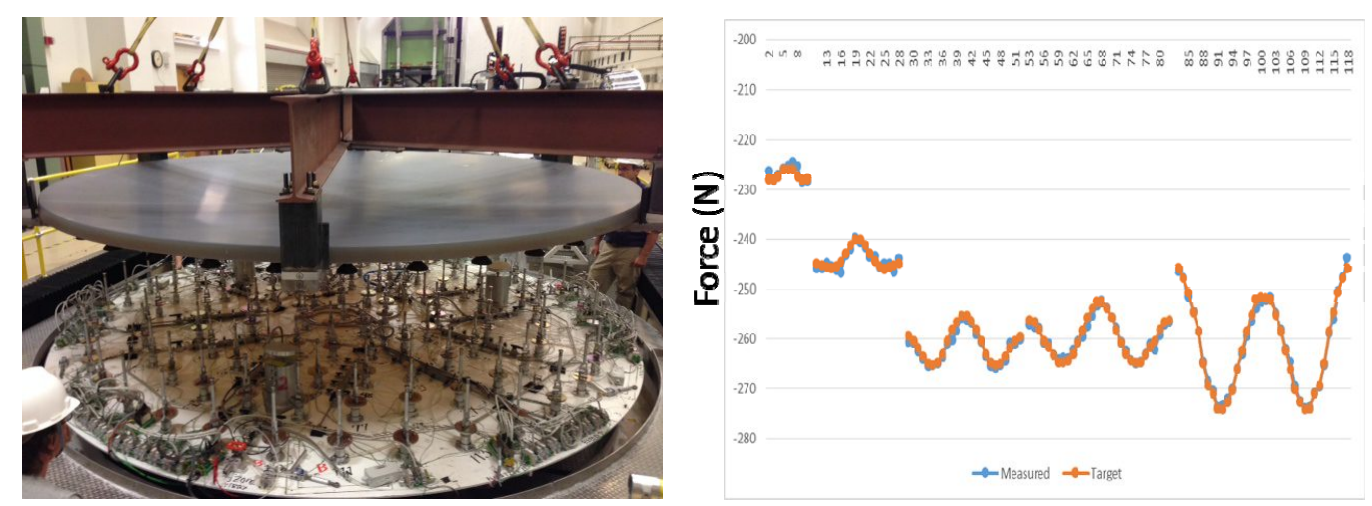

Figure 2. Mirror blank and metrology support (left) and targeted support force distribution and measured forces 
As shown in Figure 2 fabricated mirror support provides very accurate force distribution less than 1N RMS, 4N p-v.
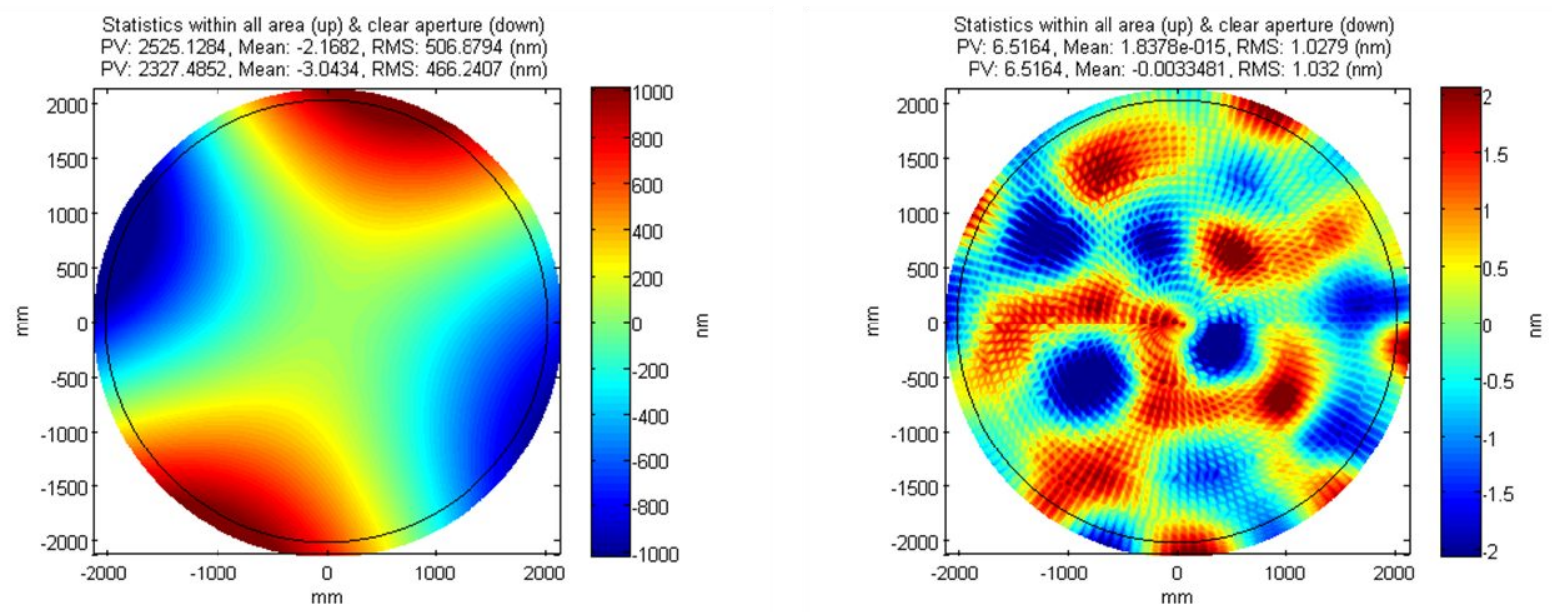

Figure 3. Mirror figure distortion due to support force error (Left) and residual after active correction (Right)

To evaluate the effect of the force difference between the target and measurement a finite element model is used. Using the model and measured force difference the surface figure is expected to be distorted as in Figure 3 in left and the residual figure after applying 30 bending mode correction is estimated as in Figure 3 in right. The residual surface and the correction force are 1nm RMS and 1N RMS respectively and both are well within tolerable range.

\section{FABRICATION PROCESS}

The mirror fabrication process is summarized in Table 3 from the generated blank to the final figuring. Metrology used for each processing phase is also listed with a redundant testing to ensure the result. All data processing and generating processing runs are done a custom optimization algorithm developed by the University of Arizona.

Table 3. Fabrication process of $4.2 \mathrm{~m}$ off-axis primary mirror of DKIST

\begin{tabular}{|c|c|}
\hline Generated Surface \\
$-\quad$ As delivered status: D64 Diamond Wheel \\
$-\quad$ Inspection \\
$\quad$ Rough Grinding \\
$-\quad$ Loose abrasive grinding with 40um Alumina \\
$-\quad$ Various size passive Tools \\
$-\quad$ Laser Tracker Surface Scan and Large Spherometer \\
Fine Grinding \\
$-\quad$ Loose abrasive grinding with 25-12um Alumina \\
$-\quad$ Various size passive tools \\
$-\quad$ IR SCOTS surface measurement and Laser Tracker \\
Polish out \\
$-\quad$ Cerium Oxide polishing \\
$-\quad$ SCOTS surface measurement and Laser Tracker \\
Figuring \\
$-\quad$ Cerium Oxide polishing \\
$-\quad$ Various size passive tools \\
\end{tabular}


Computer Controlled Fabrication system has been used for entire DKIST mirror processing. Newly developed multi-axis fabrication system with $600 \mathrm{~mm}$ active Stressed lap and 300mm passive FLEX lap are shown in Figure 4. The multi-axis computer controlled fabrication system has features of generating orbital and spinning motion and also real time feedback of the local position of the tool in the mirror coordinate. The Stressed lap is designed with 12 active bending actuators to conform the lap surface to fit to the local aspheric surface of the mirror. The passive FLEX lap is designed and optimized to the mirror figure to maximize the smoothing and figuring effect.
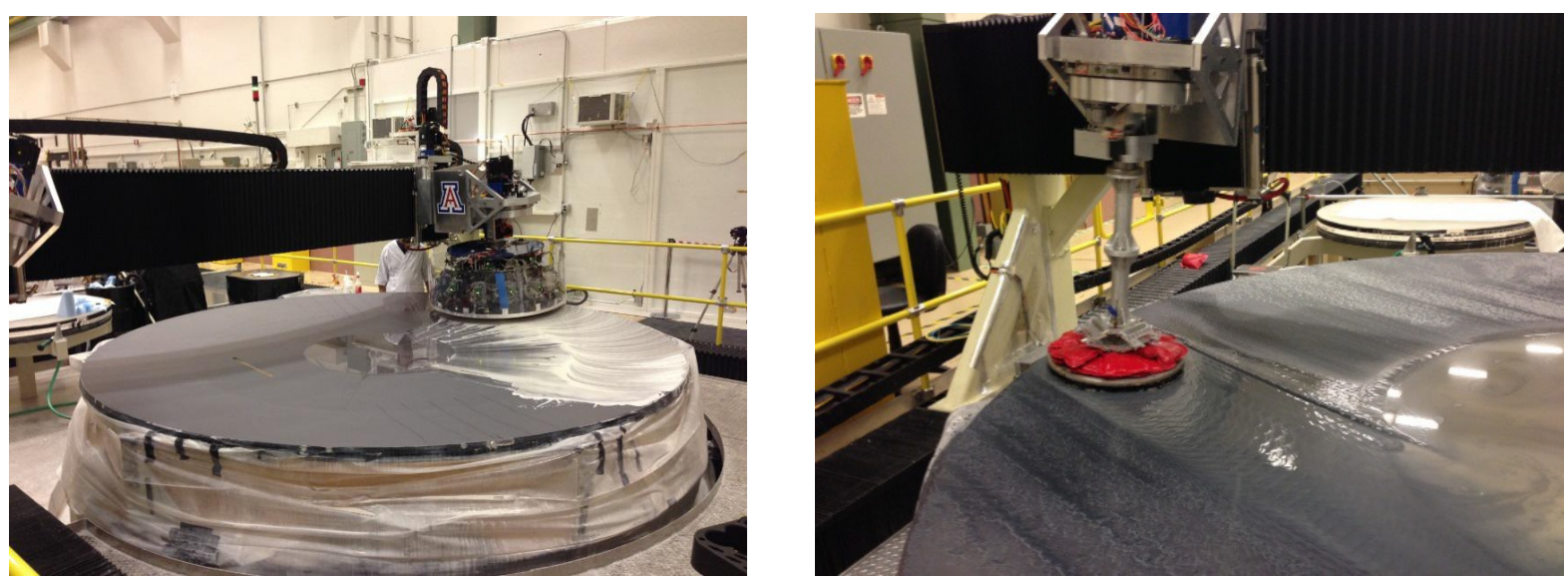

Figure 4. 600mm Active Stressed lap (Left) and 300mm FLEX lap (Right)

The fabrication schedule and convergence chart is shown in Figure 5. The total duration of the fabrication took about 13 months from the generated blank until the completion of fabrication. Thanks to the computer controlled fabrication system and well planned metrology, it was very efficient process.

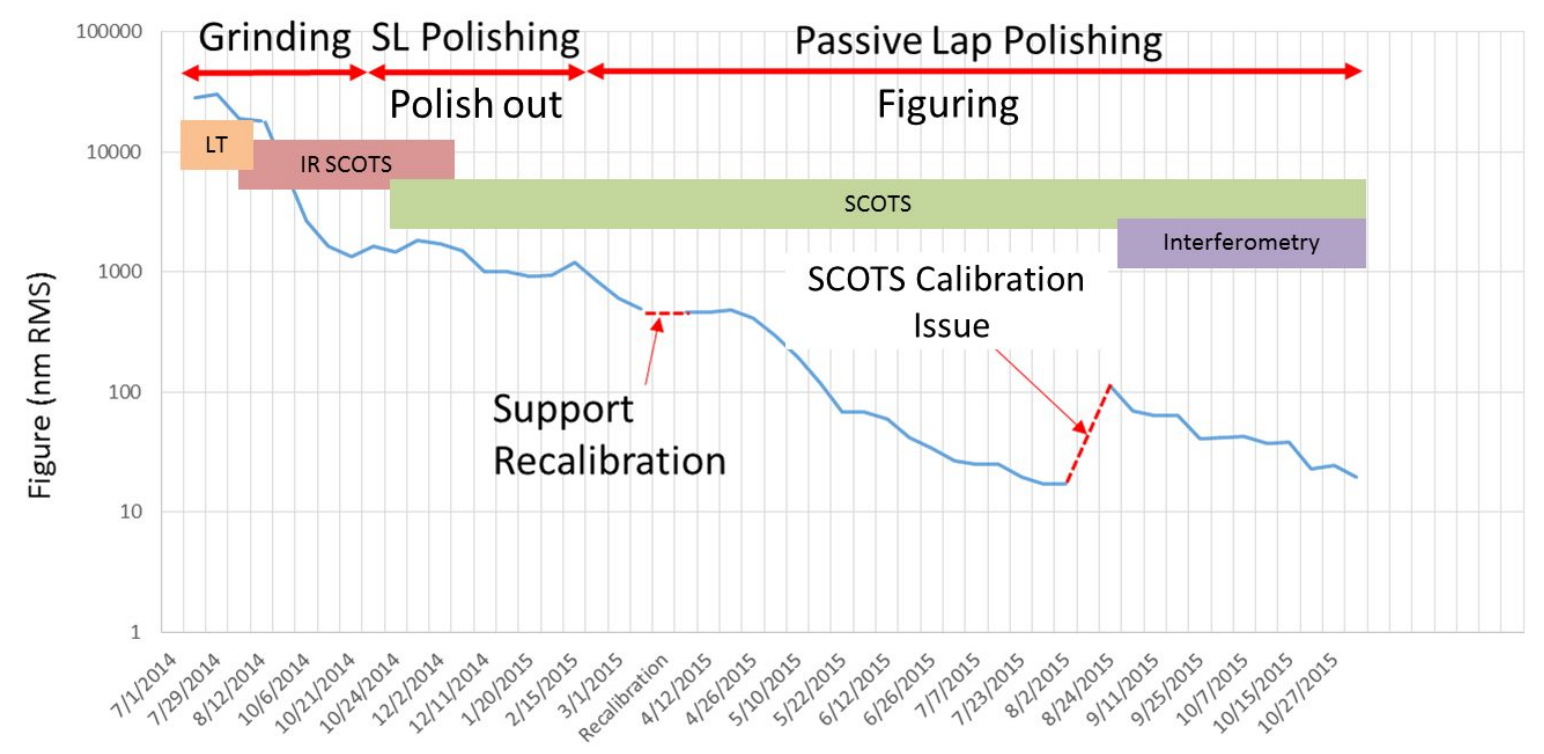

Figure 5. Fabrication schedule and convergence of DKIST primary mirror

A photo of finished DKIST primary mirror on the metrology support is shown in Figure 6. The multi-axis computer controlled fabrication system is also shown in behind of the mirror. 


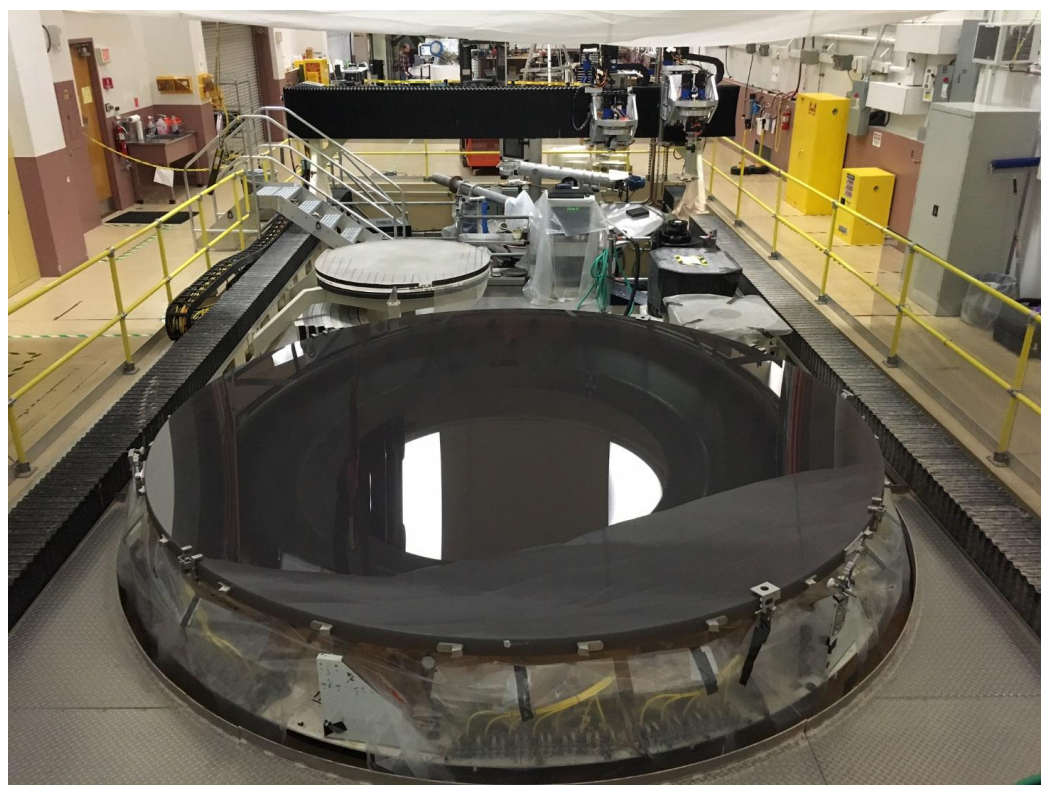

Figure 6. Photo of finished DKIST primary mirror

\section{METROLOGY}

The surface figure metrology is planned to cover the required spatial range covering the full aperture, mid spatial frequency feature and surface finish. Additionally BRDF requirement is set to specify the scattering.

For the full aperture surface metrology CGH null interferometry is performed for the principal test and Visible SCOTS test is used to guide fabrication as well as a redundant test. Figure 7 shows error budget of the principal optical test.

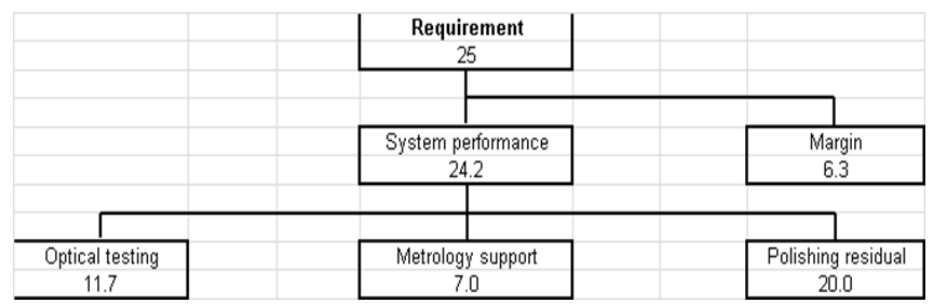

\begin{tabular}{l|} 
Approximate budget for optical testing \\
$\begin{array}{l}\text { Effect of fold sphere calibration } \\
\text { Calibration of interferometer, CGH } \\
\text { wavefront }\end{array}$ \\
$\begin{array}{l}\text { Residual from alignment, after active } \\
\text { correction }\end{array}$ \\
$\begin{array}{l}\text { Margin } \\
\text { RSS }\end{array}$ \\
\hline
\end{tabular}

Figure 7. Surface metrology error budget

Subaperture deflectometry and micro finish topography are used to evaluate mid-spatial frequency feature and surface finish. The details of metrology have been discussed in the following sections.

\subsection{Visible Deflectometry - SCOTS}

The SCOTS technique works for measuring aspheric optics without requiring additional optics. We illustrate the dynamic range of the technique using the reverse ray trace interpretation of the test where the camera aperture is replaced with a point source. The reflected light from this point source from the aspheric mirror under test would intercept the display and create an illumination pattern. The size of the display must be large enough to accommodate all of the ray bundles as defined by this reverse ray trace. If the display can be located near the center of curvature, then this 
size is approximately equal to twice the product of the aspheric slope (the gradient of the aspheric departure) and the radius of curvature. 3,4

For SCOTS test of DKIST primary mirror, two cameras and one large LCD display are configured as in Figure 8 at near the center of curvature. The display was selected to illuminate the mirror with phase shifting. Two camera provides simultaneous deflected image acquisition. SCOTS test has been performed 8 different positions of the mirror. With the configuration total of 16 measurements were made and averaged.
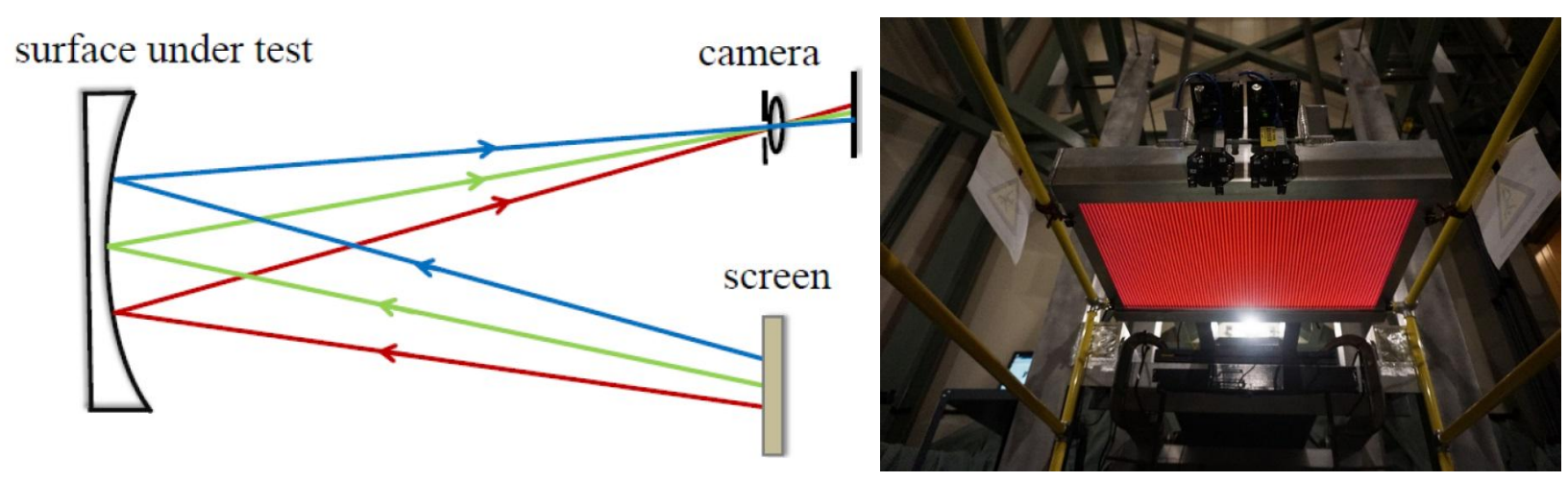

Figure 8. Concept of SCOTS test (Left) and actual SCOTS test configuration of DKIST primary mirror (Right)
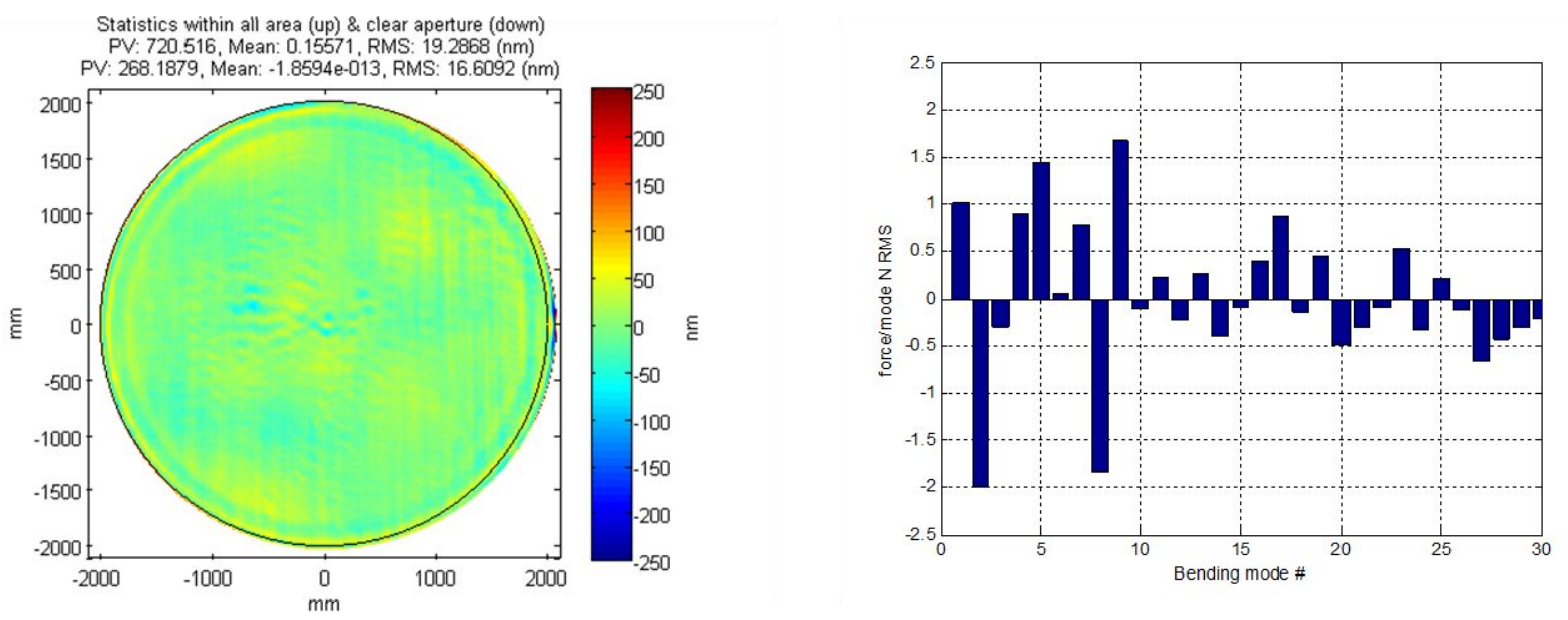

Figure 9. Surface measurement (Left) and modal correction force (right) from Visible SCOTS

The final result from Visible SCOTS test is presented in Figure 9.

\subsection{IR Deflectometry ${ }^{3,5}$}

The SCOTS technique requires specular reflection from the surface, and we desire measurements of ground glass surfaces that are diffuse for visible wavelength light. We achieve these measurements by utilizing a longer wavelength that reflects from these rough surfaces. We use thermal infrared radiation from a heated tungsten filament as the source, and a thermal imager that uses a microbolometer array as the camera. We call this system SLOTS, the Scanning Longwave Optical Test System. We performed measurements to evaluate the reflectivity of a thermal source as a function of grit size for the grinding of the surface. We get adequate reflectance for fine ground surfaces made using loose abrasives as large as $25 \mu \mathrm{m}$. 

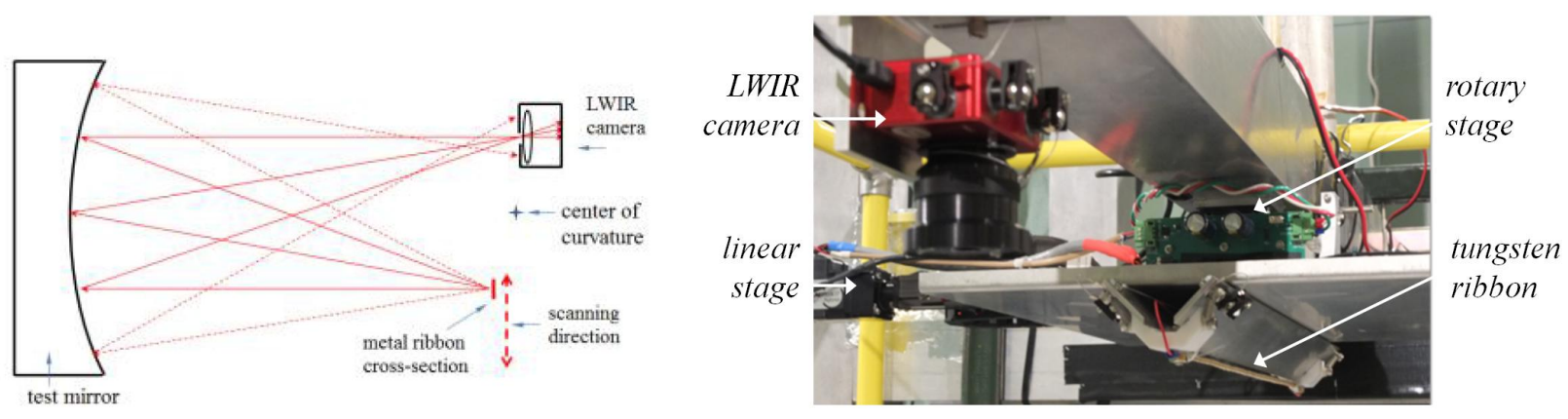

Figure 10. Concept of IR SCOTS test (Left) and actual SCOTS test configuration of DKIST primary mirror (Right)

The IR SCOTS test was very efficient metrology to guide the mirror fabrication process down to approximately $1 \mu \mathrm{m}$ RMS surface with 25-12 $\mu \mathrm{m}$ grit loose abrasive grinding.

\subsection{Principal Metrology - CGH Null Interferometry}

The interferometry approach for DKIST primary mirror is similar to optical testing performed on two other large mirrors - primary mirrors of NST and GMT - fabricated at the University of Arizona. A layout of the null test is in Figure 11. A large fold sphere is used to compensate for most of the astigmatism generated by testing the off-axis primary mirror at its center of curvature. The residual aberration is corrected using a computer generated hologram (CGH). Careful alignment and calibration of these components is required.

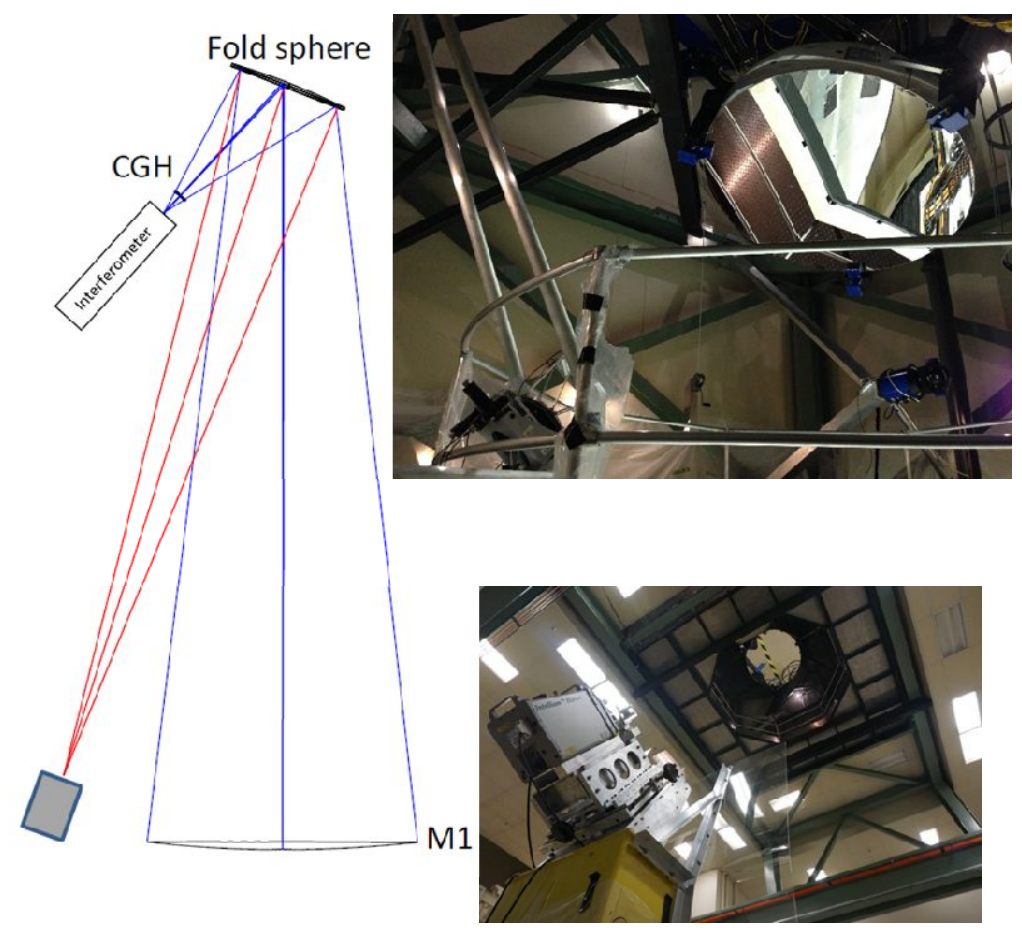

Figure 11. Construction of the interferometry 
The tolerances established for the test are shown in Table 4. The Interferometer Assembly (IA) consists of a 4D PhaseCam 6000 Interferometer and the CGH. Critical alignments are measured using a FARO laser tracker. Sphere Mounted Retroreflectors (SMRs) for the tracker measurements are attached to the CGH, large fold sphere, and the primary mirror (the latter on removable fixtures placed at the four compass points)..

Table 4. Alignment tolerance of the CGH null interferometry of DKIST

\begin{tabular}{cc}
\hline Parameter & Tolerance \\
\hline CGH to Interferometer Tilts & $1 / 3$ Fringes \\
CGH to Interferometer Focus & $1 / 4$ Fringes \\
IA Decenters (X, Y) & $50 \mu \mathrm{m}$ \\
IA Focus (Z) & $50 \mu \mathrm{m}$ \\
IA Tilt(X), Tilt(Y) & $0.001^{\circ}$ \\
IA Tilt(Z) & $0.01^{\circ}$ \\
Large Fold Sphere Tilts & $0.0014^{\circ}$ \\
Large Fold Sphere Defocus & $30 \mu \mathrm{m}$ \\
Primary Mirror Tilts & 5 Fringes \\
\hline
\end{tabular}

The large fold sphere is a $1.8 \mathrm{~m}$ mirror mounted to a hexapod. An ESDI Intellium H2000 interferometer was mounted at the center of curvature of this mirror, providing a measurement of its figure error that is subtracted from the measurement of the primary. SMRs are mounted directly to the mirror surface, allowing use of a laser tracker to accurately align the mirror using its hexapod.

The 9" CGH contains the null pattern, an alignment pattern, fiducial patterns, small Fresnel lens patterns, and a chrome patch as in Figure 12. The alignment pattern is an annular zone whose fringes are used to align the CGH to the interferometer. The fiducial patterns project spots onto the edges of the primary mirror, along the symmetry axis. The small Fresnel Zone Plate patterns are used to accurately align SMRs that are bonded to the CGH surface. The diameter of the CGH provides an insufficient baseline for the laser tracker to support the $0.001^{\circ}$ tilt tolerance on the CGH. This tolerance is achieved using the reflective chrome patch to measure the mirror image of an SMR.

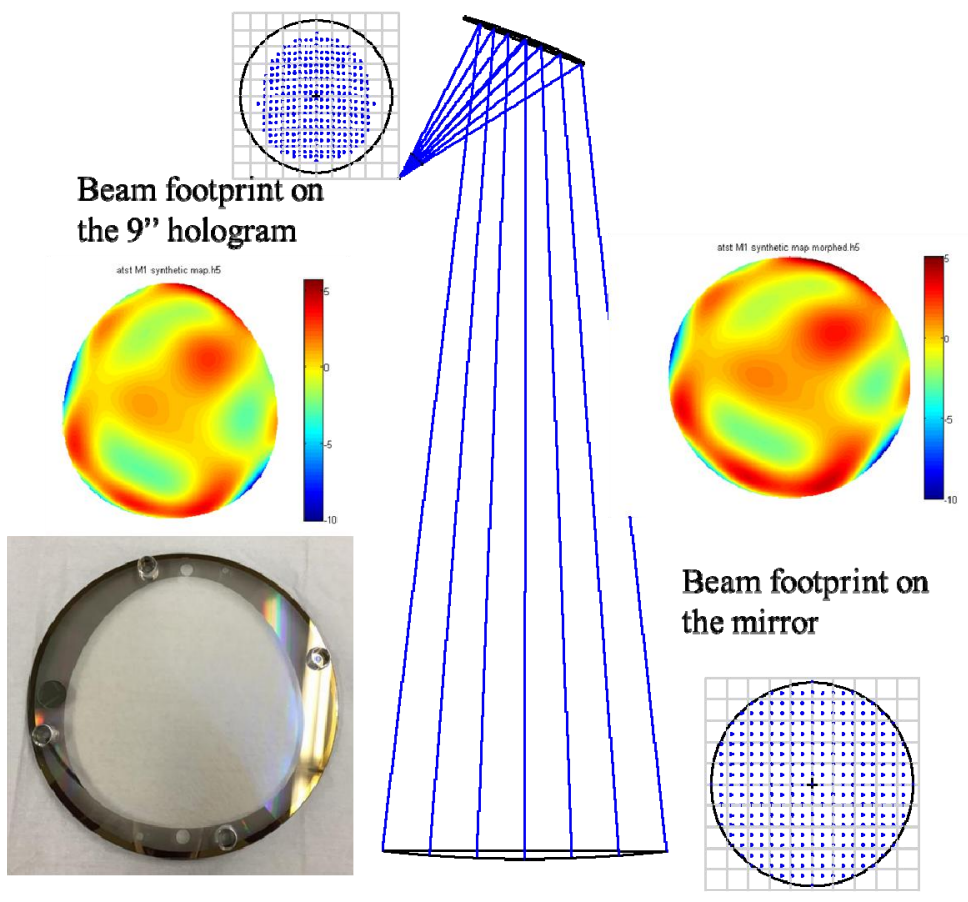

Figure 12. Surface figure distortion and Computer Generated Hologram (CGH) 
The test configuration puts significant mapping distortion into the beam as shown in Figure 12. This must be removed to create an accurate surface map in coordinates on the primary mirror, as well as for subtracting errors from the large fold sphere from the primary interferometer measurement. We use orthogonal mapping polynomials to morph the maps. Fiducials are placed on the primary mirror and measured using a laser tracker. The $4 \mathrm{D}$ interferometer measurement is morphed into the primary mirror coordinates using data from a ZEMAX ray trace.
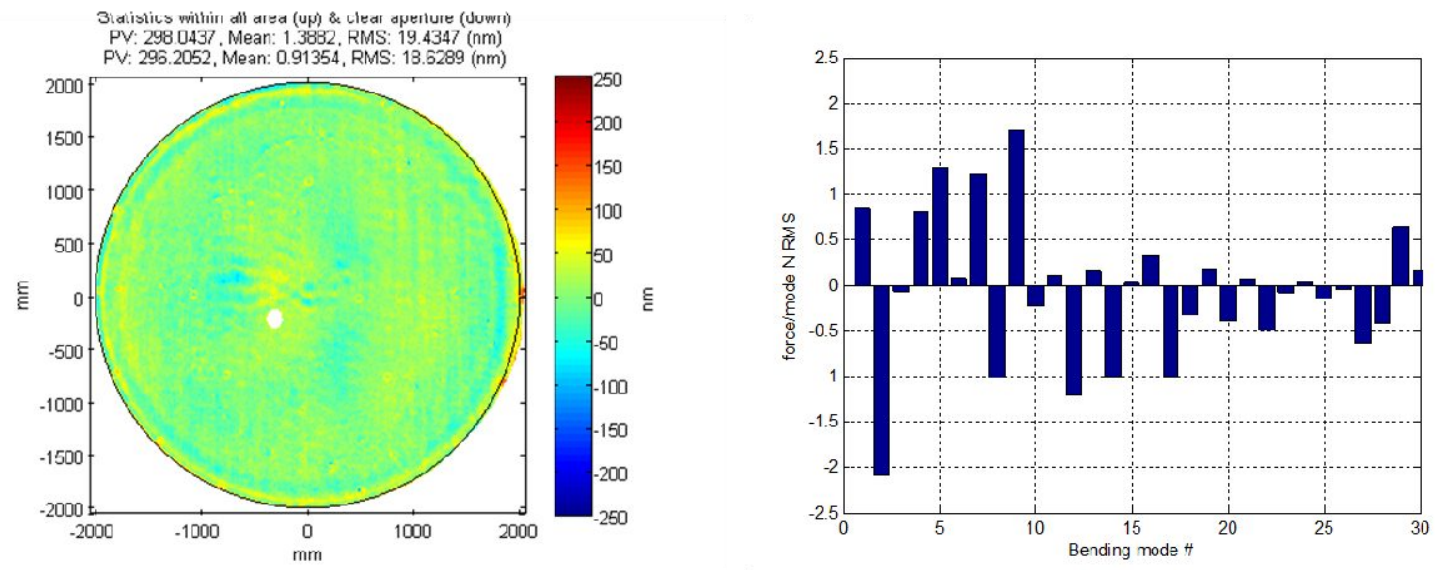

Figure 13. Surface measurement (Left) and modal correction force (right) from CGH null interferometry

The final measurement from CGH null interferometry of DKIST primary mirror is shown in Figure 13.

\subsection{Subaperture Deflectometry - SPOTS}

SPOTS is a portable SCOTS system that uses a micro-display and collimating lens that can be set on top of a large mirror to measure high frequency surface irregularities from 10 to 1000 cycles $/ \mathrm{m}$. The layout of the test system is shown below. This system measures to sub-nm precision over a $125 \mathrm{~mm}$ diameter region. The dynamic range provides the ability to measure aspheric surfaces, which gives SPOTS a distinct advantage over interferometry. Also, this system can be refocused to measure surfaces with wide range of curvatures from concave through flat to convex. ${ }^{3}$
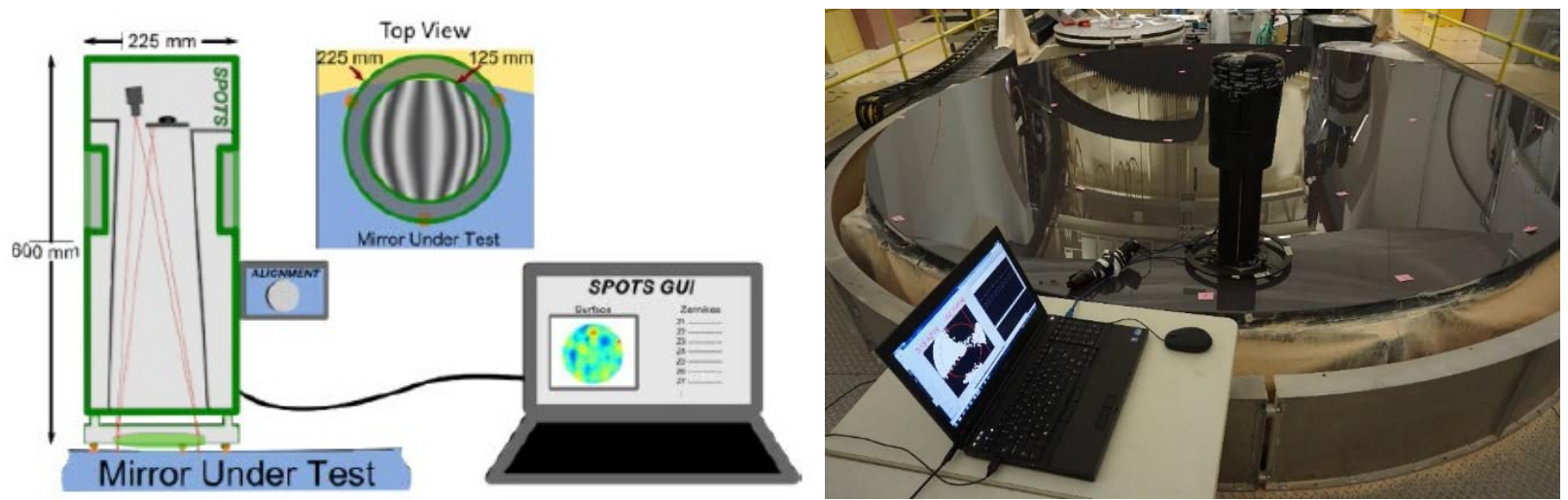

Figure 14. System configuration of SPOTS (Left) and photo of SPOTS measurement on the DKIST mirror

The actual test configuration and photo taken from the measurement are shown in Figure 14. The measured data are presented in Figure 15 and the result shows 3.6-5.2 nm RMS over $125 \mathrm{~mm}$ circular aperture. 

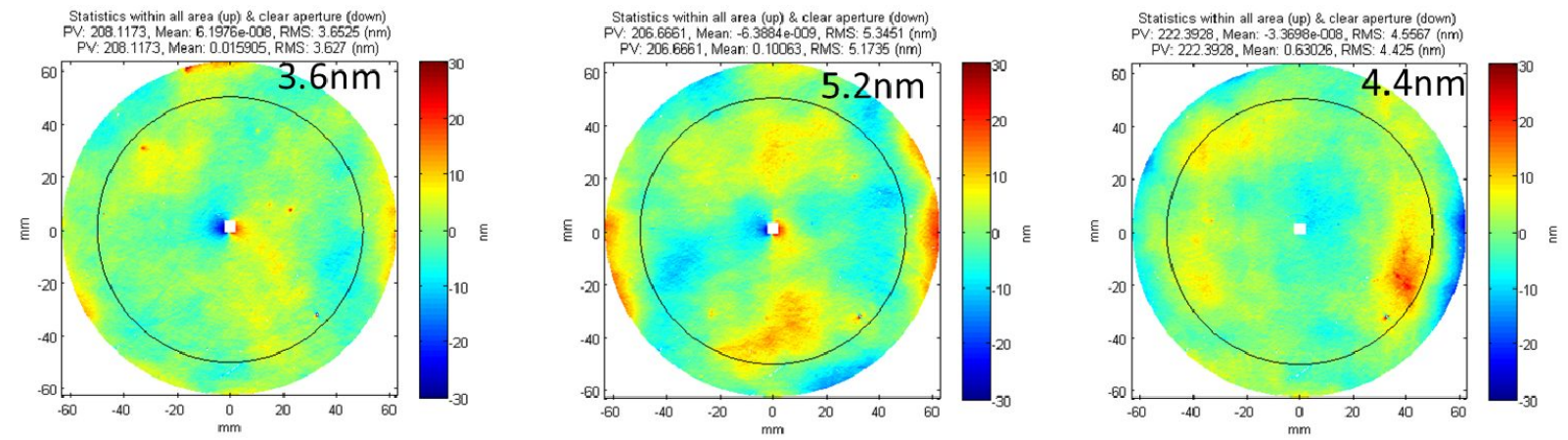

Figure 15. SPOTS measurements of DKIST mirror

\subsection{Surface Finish and BRDF}

The surface finish of DKIST primary mirror has been evaluated by measuring the surface roughness using the Micro Surface Topography(MFT) apparatus as in Figure 16. With 2.5x Nikon Mirau objective and 1024x764 pixel camera the measurement covers approximately $3 \mathrm{~mm} \times 2.5 \mathrm{~mm}$ rectangular aperture with $3 \mu \mathrm{m}$ spatial resolution. The measured surface roughness was approximately $1 \mathrm{~nm}$ RMS as in Figure 17.

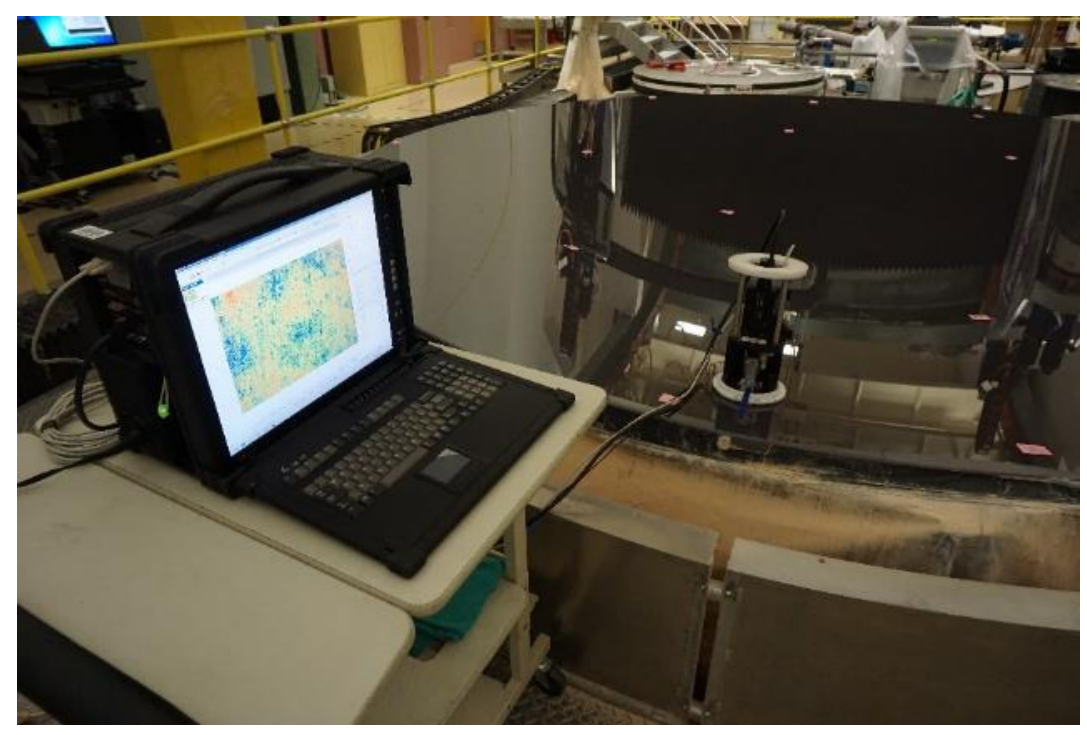

Figure 16. Photo of surface finish measurement using Micro Finish Topography apparatus
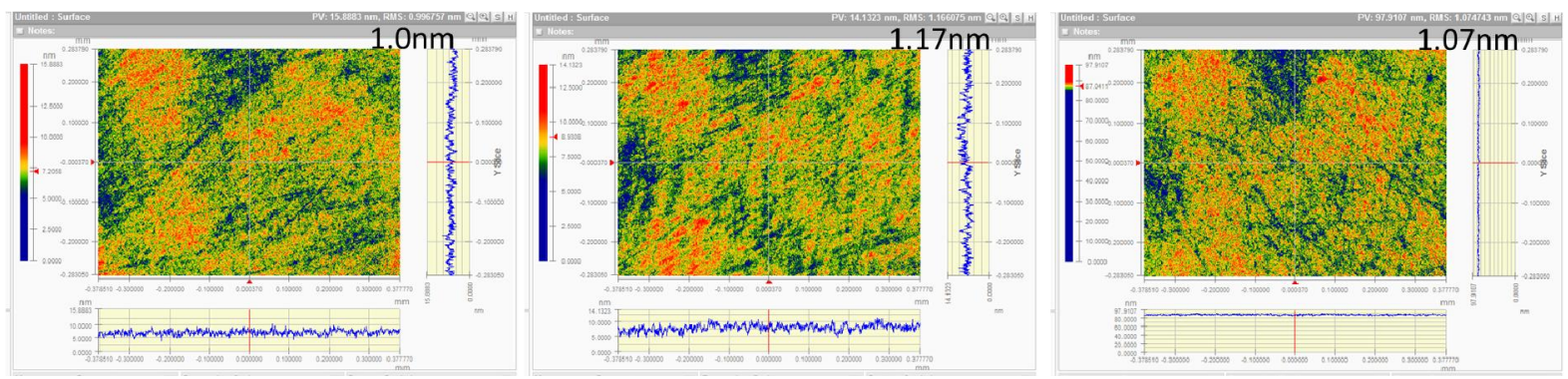

Figure 17. Surface finish measurements 
In addition to the surface roughness, the requirement for Bidirectional Reflectance Distribution Function (BRDF) was specifically set for the mirror. The BRDF of the finished DKIST mirror was evaluated using PSD by converting BRDF using Rayleigh-Rice theory. ${ }^{6}$ As in Figure 18 the measured BRDF meeting specification very well.

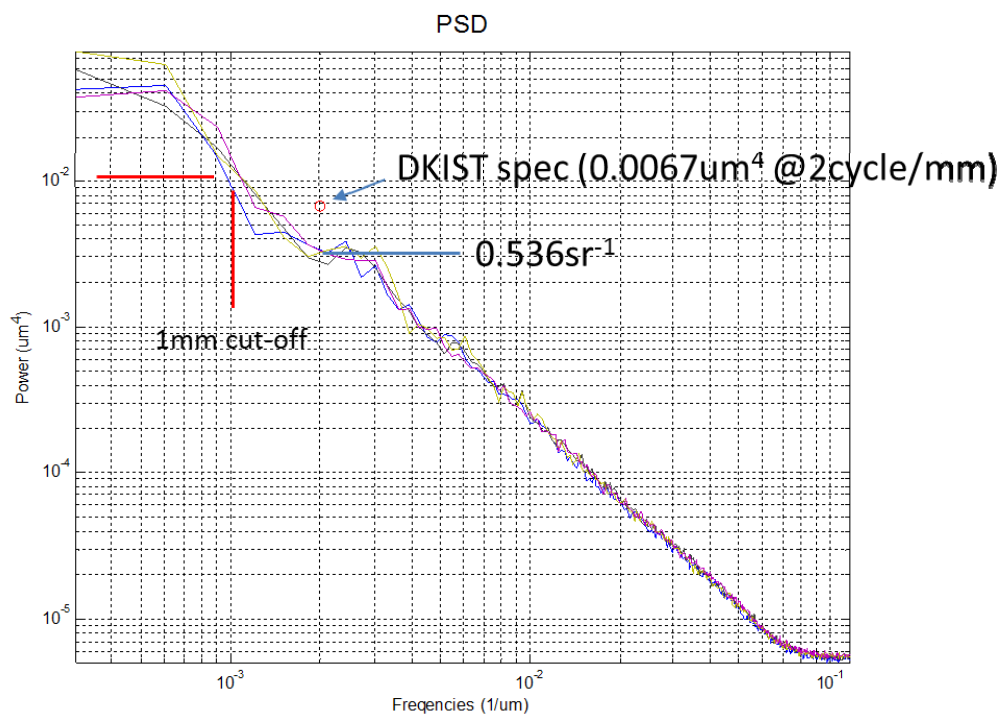

Figure 18. Power Spectrum Density (PSD) plot with BRDF specification and performance

\section{CONCLUDING REMARKS}

Fabrication of the $4.2 \mathrm{~m}$ off-axis aspheric primary mirror of DKIST has been successfully completed and the summary of the finished mirror performance is shown in the Table 4 . The mirror meets every aspect of the specification and requirement. UA team finished the mirror to 10 Angstrom roughness. Typical mirrors are finished with 20 Angstrom and this causes a small amount of scatter, which shows up as glare. With this "Super Polished" surface meets very specific $\mathrm{BRDF}$ (scatter) requirement. Maybe, no other large optics have been made to such precision.

Table 4. Summary of fabrication performance of $4.2 \mathrm{~m}$ off-axis primary mirror of DKIST

\begin{tabular}{lcc}
\hline Items & Requirement & Delivered value \\
\hline Radius of curvature for off-axis paraboloid (OAP) & $16000 \pm 15 \mathrm{~mm}$ & $16000 \pm 0.08 \mathrm{~mm}$ \\
Off axis distance with respect to ideal OAP & $4000 \pm 2 \mathrm{~mm}$ & $4000 \pm 0.18 \mathrm{~mm}$ \\
Clocking angle with respect to ideal OAP & $<2 \mathrm{arcmin}$ & $0 \pm 0.052 \mathrm{arcmin}$ \\
Surface irregularity over full aperture & $<25 \mathrm{~nm} \mathrm{rms}$ & $<18.8 \mathrm{~nm} \mathrm{rms}$ \\
High frequency surface errors $(1-100 \mathrm{~mm}$ period) & $<8 \mathrm{~nm} \mathrm{rms}$ & $5.14 \pm 1.2 \mathrm{~nm} \mathrm{rms}$ \\
Surface roughness $(<1 \mathrm{~mm}$ period) & $>20 \AA \mathrm{rms}$ & $10.5 \pm 1.1 \AA \mathrm{rms}$ \\
BRDF at 0.002 radians from specular & $<1.0 \mathrm{sr}^{-1}$ & $0.568 \pm 0.193 \mathrm{sr}^{-1}$ \\
Combined area for all surface imperfections & $<200 \mathrm{~mm}^{2}$ & $58.9 \mathrm{~mm}^{2}$ \\
Maximum actuator force required for active correction & $<20 \mathrm{~N}$ & $10.9 \mathrm{~N}$ \\
Clear aperture diameter projected onto off axis mirror & $4.000 \mathrm{~m}$ & $4.000 \mathrm{~m}$ \\
\hline
\end{tabular}




\section{ACKNOWLEDGEMENTS}

We thank to DKIST primary mirror fabrication project team including Dr. Chen Liang and Dr. Joseph McMullin in NSO and also thank to Optical Engineering and Fabrication Facility at College of Optical Sciences including Mr. Jeffrey Kingsley, Mr. Bruce Cook, Mr. Daniel Caywood, Mr. Logan Graves and many others who supported the project behind the scenes.

\section{REFERENCES}

[1] J. P. McMullin et al., "The Advanced Technology Solar Telescope: design and early construction," Proc. SPIE 8444,844407 (2012).

[2] National Solar Observatory, [Advanced Technology Solar Telescope], (http://dkist.nso.edu/sites/atst.nso.edu/files /press/ATST book.pdf), 2014

[3] J. H. Burge, P. Su, G. Butel, R. Huang, A. Maldonado, T. Su, "Measuring large mirrors using SCOTS, the Software Configurable Optical Test System", Proc. SPIE 9151, (2014)

[4] Tianquan Su, Won Hyun Park, Robert E. Parks, Peng Su, James H. Burge, "Scanning Long-wave Optical Test System - a new ground optical surface slope test system", Proc. SPIE 8126, (2011).

[5] Peng Su, Manal Khreishi, Run Huang, Tianquan Su, and James H. Burge, "Precision aspheric optics testing with SCOTS: a deflectometry approach", Proc. SPIE 8788, (2013).

[6] Kashmira Tayabaly, John C. Stover, Robert E. Parks, Matthew Dubin, James H. Burge, "Use of the surface PSD and incident angle adjustments to investigate near specular scatter from smooth surfaces", Proc. SPIE 8838, Optical Manufacturing and Testing X, 883805 (September 7, 2013); doi:10.1117/12.2024612. 